PROCEEDINGS OF THE

AMERICAN MATHEMATICAL SOCIETY

Volume 133, Number 8, Pages 2267-2272

S 0002-9939(05)07407-1

Article electronically published on March 4, 2005

\title{
FREE GROUP FACTORS AND FACTORS WITH SOME DECOMPOSITIONS
}

\author{
JUNHAO SHEN AND XIAOXIA ZHANG
}

(Communicated by David R. Larson)

\begin{abstract}
In this paper, we show that if type $I I_{1}$ von Neumann factors $\mathcal{M}$ have some decompositions introduced by Liming Ge and Sorin Popa, then these von Neumann factors are not isomorphic to free group factors $L\left(F_{n}\right)(n \geq 2)$. Thus we have proved the number $l_{a}$ defined by Ge and Popa bigger than 3 for all free group factors and we also extend some results of M. Stefan.
\end{abstract}

\section{INTRODUCTION}

In studying the structure of type $I I_{1}$ factors, Connes and Jones [1] proved that von Neumann algebras with property $\mathrm{T}$ are not isomorphic to free group factors $L\left(F_{n}\right)(n \geq 2)$. By introducing free entropy in 8] and 9], Voiculescu proved that free group factors $L\left(F_{n}\right)$ are not isomorphic to von Neumann algebras that have Cartan subalgbras. Also using Voiculescu's free entropy, Liming Ge 2, 3] proved that free group factors possess no simple masa and are prime. For the free group factors, Voiculescu [7] proved the following result.

Proposition 1. Let $L\left(F_{n}\right)(n>1)$ be free group factors. Then

$$
\left(L\left(F_{n}\right)\right)_{1 / k} \cong L\left(F\left(n k^{2}-k^{2}+1\right)\right),
$$

where $k$ is a positive integer.

In the study of von Neumann algebras, S. Popa 5 found a large class of nonhyperfinite type $I I_{1}$ factors (called thin factors) having a decomposition of the form $M=\overline{s p} R_{0} R_{1}$, with $R_{0}, R_{1}$ hyperfinite $(\overline{s p} X$ denotes the closed linear span of the set $X$ in the Hilbert-space norm given by the trace of the ambient type $I I_{1}$ factor). Liming Ge and Sorin Popa 4 proved that thin factors are not isomorphic to free group factors $L\left(F_{n}\right)(n \geq 3)$. In the same paper [4], Liming Ge and Sorin Popa also proved that if a type $I I_{1}$ factor $\mathcal{M}$ has an irreducible hyperfinite quasiregular subfactor $\mathcal{R}_{0} \subset \mathcal{M}$ (that is, so that $\mathcal{R}_{0}^{\prime} \cap\left\langle\mathcal{M}, \mathcal{R}_{0}\right\rangle$, where $\left\langle\mathcal{M}, \mathcal{R}_{0}\right\rangle$ denotes the von Neumann algebra generated in $B\left(L^{2}(\mathcal{M}, \tau)\right)$ by $\mathcal{M}$ and by the orthogonal projection $e_{\mathcal{R}_{0}}$ of $L^{2}(\mathcal{M}, \tau)$ onto $L^{2}\left(\mathcal{R}_{0}, \tau\right)$, is generated by projections of finite trace in $\left.\left\langle\mathcal{M}, \mathcal{R}_{0}\right\rangle\right)$, then from [4] there exist an abelian subalgebra $\mathcal{A} \subset \mathcal{M}$ and an element $b \in \mathcal{M}$ such that $\mathcal{M}=\overline{\mathrm{sp}} \mathcal{R}_{0} b \mathcal{A}$. These factors have vanishing second Hochschild cohomology groups, $H^{2}(\mathcal{M}, \mathcal{M})=0$. All symmetric enveloping factors

Received by the editors June 14, 2002 and, in revised form, April 17, 2003.

2000 Mathematics Subject Classification. Primary 46L05, 47D15.

Key words and phrases. Free group factor, diffused algebra, hyperfinite $I I_{1}$ factor. 
mentioned above and all the cross-products of the hyperfinite factor by cocycle actions of a group do have this property. Hinted by Ge and Popa's work [4], Stefan [6] studied the indecomposabilty of free group factors over nonprime subfactors and abelian subalgebras and obtained the following result.

Proposition 2. If $n \geq p+2 f+2$, then the free group factor $L\left(F_{n}\right)$ does not admit a decomposition of the form

$$
\|\cdot\|_{2}-\lim _{\omega \rightarrow 0} \sum_{\substack{1 \leq j_{1}, \ldots, j_{t+1} \leq f \\ 1 \leq t \leq d}} \mathcal{M}_{j_{1}} Z^{\omega} \mathcal{M}_{j_{2}} Z^{\omega} \cdots \mathcal{M}_{j_{t}} Z^{\omega} \mathcal{M}_{j_{t+1}},
$$

where $\left\{Z^{\omega}\right\}_{\omega}$ are subsets of $L\left(F_{n}\right)$, each containing $p$ self-adjoint elements, $\mathcal{M}_{1}, \ldots$, $\mathcal{M}_{f} \subset L\left(F_{n}\right)$ are either abelian ${ }^{*}$-subalgebras or non-prime subfactors of $L\left(F_{n}\right)$, and $d \geq 1$ is an integer; and $\|\cdot\|_{2}$ denotes the $L^{2}$-norm (or trace-norm) of $L\left(F_{n}\right)$ with respect to the faithful normal tracial state $\tau$ (that is, $\|A\|_{2}^{2}=\tau\left(A^{*} A\right)$ with $\left.A \in L\left(F_{n}\right)\right)$.

In this paper, we use Proposition 1 and Proposition 2 to show that all free group factors $L\left(F_{n}\right)$ are not isomorphic to type $I I_{1}$ factors $\mathcal{M}$ with composition $\overline{s p}\left\{\mathcal{A}_{0} a_{s} \mathcal{A}_{1} b_{t} \mathcal{A}_{2}: s=1, \ldots, m, t=1, \ldots, q\right\}$ or $\overline{s p}\left\{\mathcal{A}_{0} a_{s} \mathcal{R} b_{t} \mathcal{A}_{1}: s=1, \ldots, m, t=\right.$ $1, \ldots, q\}$ or $\overline{s p} \mathcal{R}_{0} \mathcal{R}_{1}$, where $\mathcal{A}_{0}, \mathcal{A}_{1}$ and $\mathcal{A}_{2}$ are diffused abelian subalgebras of $\mathcal{M}$; $\mathcal{R}, \mathcal{R}_{0}$ and $\mathcal{R}_{1}$ are hyperfinite subfactors of $\mathcal{M}$ such that $\mathcal{R}_{0}$ and $\mathcal{R}_{1}$ satisfy some conditions; and $a_{s}, b_{t}, 1 \leq s \leq m, 1 \leq t \leq q$, are selfadjoint elements in $\mathcal{M}$. As a result, type $I I_{1}$ factors with decomposition $\overline{s p} \mathcal{R} b \mathcal{A}$ are not isomorphic to all free group factors $L\left(F_{n}\right), n>1$, on $n$ generators. Our results also state that the abelian length defined in [4] of all free group factors is bigger than 3; thus we generalize some results in [6].

\section{MAIN RESUlts AND PROOF}

Von Neumann algebras are algebras of bounded operators on a Hilbert space $\mathcal{H}$, containing the identity operator $I$ and $A^{*}$ when they contain $A$ and closed in the strong-operator topology $\left(\left\{A_{n}\right\}\right.$ converges to $A$ in this topology when $\left\{A_{n} x\right\}$ converges to $A x$ for each $x \in \mathcal{H}$ ). For a von Neumann algebra $\mathcal{M}$, the commutant $\mathcal{M}^{\prime}$ of $\mathcal{M}$ consists of those operators $T$ that commute with each element of $\mathcal{M}$. Factors $\mathcal{M}$ are von Neumann algebras whose centers $\mathcal{M} \cap \mathcal{M}^{\prime}$ consist of scalar multiples of $I$. A type $I I_{1}$ factor $\mathcal{M}$ is a factor with a unique faithful tracial state $\tau$ such that $\tau(I)=1$ and $\tau(\mathcal{P}(\mathcal{M}))=[0,1]$, where $\mathcal{P}(\mathcal{M})$ is all projections in $\mathcal{M}$. If a von Neumann algebra has no minimal projections, we call such a von Neumann algebra diffused.

Let $G$ be a discrete countable group with unit $e$. For each $g \in G$, let $L_{g}$ denote the left translation of functions in the Hilbert space $l^{2}(G)$ by $g^{-1}$. Then $g \rightarrow L_{g}$ is a faithful unitary representation of $G$ on $l^{2}(G)$. Let $L(G)$ be the von Neumann algebra generated by $\left\{L_{g}: g \in G\right\}$. In general, $L(G)$ is a finite von Neumann algebra. It is a factor (of type $I I_{1}$ ) precisely when each conjugacy class in $G$ (other than that of $e$ ) is infinite, that is to say, $G$ is an i.c.c. group.

Specific examples of such $I I_{1}$ factors result from choosing for $G$ any of the free groups $F_{n}$ on $n$ generators $(n \geq 2)$. We shall prove that none of the $I I_{1}$ factors $L\left(F_{n}\right)$ are isomorphic to three classes of type $I I_{1}$ factors with some decompositions. 
Theorem 2.1. Let $\mathcal{M}$ be a type $I I_{1}$ factor with the decomposition

$$
\mathcal{M}=\overline{s p}\left\{\mathcal{A}_{1} a_{s} \mathcal{A}_{2} b_{t} \mathcal{A}_{3}: s=1, \ldots, m, t=1, \ldots, q\right\},
$$

where $\mathcal{A}_{j}, j=1,2,3$, are diffused abelian subalgebra of $\mathcal{M}$ and $a_{s}, b_{t}$ are non-zero elements in $\mathcal{M}$, then $\mathcal{M}$ is not isomorphic to any one of the free group factors $L\left(F_{n}\right)(n>1)$.

Proof. Since $\mathcal{A}_{2}$ is diffused, then for any $k$ in $\mathbf{N}$, one can choose a projection $p \in \mathcal{A}_{2}$ such that $\tau(p)=\frac{1}{k}$. So there exist unitary elements $u$ and $v$ in $\mathcal{M}$ satisfying $u p u^{*} \in \mathcal{A}_{1}$ and $v p v^{*} \in \mathcal{A}_{3}$. Also, we have

$$
\begin{aligned}
& \mathcal{M}=\overline{s p}\left\{u^{*} \mathcal{A}_{1} u u^{*} a_{s} \mathcal{A}_{2} b v v^{*} \mathcal{A}_{3} v: 1 \leq s \leq m, 1 \leq t \leq q\right\} \\
& =\overline{s p}\left\{u^{*} \mathcal{A}_{1} u u^{*} a_{s} \mathcal{A}_{2} b_{t} v v^{*} \mathcal{A}_{3} v: 1 \leq s \leq m, 1 \leq t \leq q\right\} \\
& =\overline{s p}\left\{\tilde{\mathcal{A}}_{1} u^{*} a_{s} \mathcal{A}_{2} b_{t} v \tilde{\mathcal{A}}_{3}: 1 \leq s \leq m, 1 \leq t \leq q\right\}
\end{aligned}
$$

where $\tilde{\mathcal{A}}_{1}=u^{*} \mathcal{A}_{1} u$ and $\tilde{\mathcal{A}}_{3}=v^{*} \mathcal{A}_{3} v$. We have the fact that $\tilde{\mathcal{A}}_{1}$ and $\tilde{\mathcal{A}}_{3}$ are also diffused abelian, and $p \in \widetilde{\mathcal{A}}_{1} \cap \mathcal{A}_{2} \cap \tilde{\mathcal{A}}_{3}$.

Since $\mathcal{A}_{2}$ is diffused, there exist projections $p_{j} \in \mathcal{A}_{2}$ and unitary elements $u_{j} \in$ $\mathcal{M}, j=1, \ldots, k$, such that $\sum_{j=1}^{k} p_{j}=I$ and $u_{j}^{*} p u_{j}=p_{j}$. Now

$$
\begin{aligned}
p \mathcal{M} p & =\overline{s p}\left\{p \tilde{\mathcal{A}}_{1} p u^{*} a_{s} \sum_{j=1}^{k} p_{j} \mathcal{A}_{2} b_{t} v p \tilde{\mathcal{A}}_{3} p: 1 \leq s \leq m, 1 \leq t \leq q\right\} \\
& =\overline{s p}\left\{p \tilde{\mathcal{A}}_{1} p u^{*} a_{s} \sum_{j=1}^{k} p_{j} \mathcal{A}_{2} p_{j} b_{t} v p \tilde{\mathcal{A}}_{3} p: 1 \leq s \leq m, 1 \leq t \leq q\right\} \\
& =\overline{s p}\left\{p \tilde{\mathcal{A}}_{1} p u^{*} a_{s} u_{j}^{*} p u_{j} \mathcal{A}_{2} u_{j}^{*} p u_{j} b_{t} v p \tilde{\mathcal{A}}_{3} p: j=1, \ldots, k ; 1 \leq s \leq m, 1 \leq t \leq q\right\} .
\end{aligned}
$$

Because $p \tilde{\mathcal{A}}_{1} p, p u_{j} \mathcal{A}_{2} u_{j}^{*} p$ and $p \tilde{A}_{3} p$ are abelian subalgebras of $p \mathcal{M} p$ and by Proposition 2. $p \mathcal{M} p$ is not isomorphic to $L\left(F_{n}\right)(n>6+2(m+q+1) k)$.

By Proposition 2 again, we know that $\mathcal{M}$ is not isomorphic to free group factors $L\left(F_{n}\right), n \geq 2(m+q)+8$.

If $\mathcal{M}$ is isomorphic to some one of free group factors $L\left(F_{n}\right), n<2(m+q)+8$, and if we choose a projection $p \in \mathcal{M}\left(\tau(p)=\frac{1}{k}\right)$, then $p \mathcal{M} p \cong L\left(F_{(n-1) k^{2}+1}\right)$ by Proposition 1 On the other hand, when $k$ is big enough, $(n-1) k^{2}+1>$ $6+2(s+t+1) k$, from the previous paragraphs, one has that $p \mathcal{M} p$ is not isomorphic to $L\left(F_{(n-1) k^{2}+1}\right)$. This is a contradiction. So for any one of free group factors, $L\left(F_{n}\right)$ is not isomorphic to the type $I I_{1}$ factor $\mathcal{M}$ with decomposition $\overline{s p}\left\{\mathcal{A}_{1} a_{s} \mathcal{A}_{2} b_{t} \mathcal{A}_{3}\right.$ : $1 \geq s \leq m, 1 \geq t \leq q\}$.

With the same method, one can prove the following theorem.

Theorem 2.2. Any one of free group factors $L\left(F_{n}\right), n>1$, is not isomorphic to the type $I I_{1}$ factor $\mathcal{M}$ with the decomposition $\overline{s p}\left\{\mathcal{A}_{0} a_{s} \mathcal{A}_{1}: s=1, \ldots, m\right\}$, where $\mathcal{A}_{0}$ and $\mathcal{A}_{1}$ are diffused von Neumann subalgebras and $a_{s}, s=1, \ldots, m$, are non-zero elements in $\mathcal{M}$.

Since all hyperfinite $I I_{1}$ factors are all isomorphic, and a hyperfinite $I I_{1}$ factor $\mathcal{R}$ has decomposition $\overline{s p} \mathcal{A}_{0} \mathcal{A}_{1}$ (by the irrational rotation algebra representation of $\mathcal{R})$, then as a corollary we have the following result.

Corollary 2.3. None of free group factors $L\left(F_{n}\right), n>1$, is isomorphic to the type $I I_{1}$ factor $\mathcal{M}$ with the decomposition $\overline{s p}\left\{\mathcal{R} a_{s} \mathcal{A}: s=1, \ldots, m\right\}$, where $\mathcal{R}$ is a hyperfinite subfactor of $\mathcal{M}$ and $\mathcal{A}$ are diffused von Neumann subalgebras and $a_{s}, s=1, \ldots, m$, are non-zero elements in $\mathcal{M}$. 
Theorem 2.4. None of free group factors $L\left(F_{n}\right), n>1$, is isomorphic to a type $I I_{1}$ factor $\mathcal{M}$ with the decomposition $\overline{s p}\left\{\mathcal{A}_{0} a_{s} \mathcal{R} b_{t} \mathcal{A}_{1}: s=1, \ldots, m, t=1, \ldots, q\right\}$, where $\mathcal{A}_{0}$ and $\mathcal{A}_{1}$ are diffused von Neumann subalgebras, $\mathcal{R}$ is a hyperfinite subfactor of $\mathcal{M}$ and $a_{s}, b_{t}, s=1, \ldots, m ; t=1,2, \ldots, q$, are non-zero elements in $\mathcal{M}$.

Proof. By Proposition 2, we know that the factor $\mathcal{M}$ in Theorem 2.4 is not isomorphic to free group factors $L\left(F_{n}\right), n \geq 2(m+q)+8$.

On the other hand, pick $p \in \mathcal{M}$ and $\tau(p)=\frac{1}{k}$. Then there exist unitary elements $u_{0}, u_{1}$ and $u_{R}$ in $\mathcal{M}$ such that $u_{0}^{*} p u_{0} \in \mathcal{A}_{0}, u_{1}^{*} p u_{1} \in \mathcal{A}_{1}$ and $u_{R}^{*} p u_{R} \in \mathcal{R}$. We also know that

$$
\begin{aligned}
& \mathcal{M}=\overline{s p}\left\{u_{0} \mathcal{A}_{0} u_{0}^{*} u_{0} a_{s} u_{R}^{*} u_{R} \mathcal{R} u_{R}^{*} u_{R} b_{t} u_{1}^{*} u_{1} \mathcal{A}_{1} u_{1}^{*}: s=1,2, \ldots, m ; t=1,2, \ldots, q\right\} \\
& =\overline{s p}\left\{\tilde{\mathcal{A}}_{0} \tilde{a}_{s} \tilde{\mathcal{R}} \tilde{b}_{t} \tilde{\mathcal{A}}_{1}: 1 \leq s \leq m, 1 \leq t \leq q\right\}
\end{aligned}
$$

where $\tilde{\mathcal{A}}_{i}=u_{i} \mathcal{A}_{i} u_{i}^{*}, i=0,1$, and $\tilde{\mathcal{R}}=u_{R} \mathcal{R} u_{R}^{*}$. So $p \in \tilde{\mathcal{A}}_{0} \cap \tilde{\mathcal{R}} \cap \tilde{\mathcal{A}}_{1}$. Then we assume that $\mathcal{M}=\overline{s p}\left\{\mathcal{A}_{0} a_{s} \mathcal{R} b_{t} \mathcal{A}_{1}: 1 \leq s \leq m, 1 \leq t \leq q\right\}$ and there is a projection $p \in \mathcal{A}_{0} \cap \mathcal{R} \cap \mathcal{A}_{1}$ such that $\tau(p)=\frac{1}{k}$, where $a_{s}, 1 \leq s \leq m$, and $b_{t}, 1 \leq t \leq q$, are elements in $\mathcal{M}$ (not necessary self-adjoint elements).

Since $\mathcal{R}$ is a type $I I_{1}$ factor, then there are orthogonal projections $q_{j} \in \mathcal{A}(j=$ $1, \ldots, k)$ and unitary elements $v_{j} \in \mathcal{R}(j=1, \ldots, k)$ such that $\sum_{j=1}^{k} p_{j}=I, \tau\left(p_{j}\right)=\frac{1}{k}$ and $q_{j}=v_{j}^{*} p v_{j}$. Now

$$
\begin{aligned}
p \mathcal{M} p & =\overline{s p}\left\{p \mathcal{A}_{0} p p a_{s}\left(\sum q_{j}\right) \mathcal{R}\left(\sum q_{j}\right) b_{t} p \mathcal{A}_{1} p: 1 \leq s \leq m, 1 \leq t \leq q\right\} \\
& =\overline{s p}\left\{p \mathcal{A}_{0} p p a_{s} v_{j}^{*} p v_{j} \mathcal{R} v_{i}^{*} p v_{i} b_{t} p \mathcal{A}_{1} p: 1 \leq j, i \leq k, 1 \leq s \leq m, 1 \leq t \leq q\right\} \\
& =\overline{s p}\left\{p \mathcal{A}_{0} p p a_{s} v_{j}^{*} p \mathcal{R} p v_{i} b_{t} p \mathcal{A}_{1} p: 1 \leq j, i \leq k, 1 \leq s \leq m, 1 \leq t \leq q\right\},
\end{aligned}
$$

and $p \mathcal{A}_{0} p$ and $p \mathcal{A}_{1} p$ are also abelian subalgebras of $p \mathcal{M} p$, and $p \mathcal{R} p$ is a hyperfinite subfactor of $p \mathcal{M} p$. Then, by Proposition 2, $p \mathcal{M} p$ is not isomorphic to free group factors $L\left(F_{n}\right), n \geq 2(m+q) k+8$.

If $\mathcal{M}$ is isomorphic to one of free group factors $L\left(F_{n}\right), n<2(m+q)+8$, then $p \mathcal{M} p$ is isomorphic to $L\left(F_{(n-1) k^{2}+1}\right)$ by Proposition [ where $p$ is as above. Since $\mathcal{A}_{0}, \mathcal{A}_{1}$ and $\mathcal{R}$ are diffused subalgebras of $\mathcal{M}$, then $p$ can be chosen small enough. When $k$ is big enough, $(n-1) k^{2}+1>2(m+q) k+8$. From the second part of the above proof, $p \mathcal{M} p$ is not isomorphic to free group factors $L\left(F_{(n-1) k^{2}+1}\right)$; this is contradiction. This completes the proof.

In [4], Ge and Popa proved that free group factors $L\left(F_{n}\right), n>2$, are not isomorphic to type $I I_{1}$ factors $\mathcal{M}$ with decomposition $\overline{s p} \mathcal{R}_{0} \mathcal{R}_{1}$, where $\mathcal{R}_{0}$ and $\mathcal{R}_{1}$ are hyperfinite subfactors of $\mathcal{M}$. The following theorem states that if $\mathcal{R}_{0}$ and $\mathcal{R}_{1}$ satisfy some additional conditions, then all free group factors are not isomorphic to above type $I I_{1}$ factors.

Theorem 2.5. Let $\mathcal{M}$ be a type $I I_{1}$ factor, and let $\mathcal{M}=\overline{s p} \mathcal{R}_{0} \mathcal{R}_{1}$. If the hyperfinite subfactor $\mathcal{R}_{0} \cap \mathcal{R}_{1}$ of $\mathcal{R}_{0}$ and $\mathcal{R}_{1}$ has orthogonal projections $p_{1}, \ldots, p_{s}$ such that $p_{1}+\cdots+p_{s}=I$ and $\max _{1 \leq j \leq s}\left\{\tau\left(p_{j}\right)\right\}<\frac{1}{2}$, then all free group factors $L\left(F_{n}\right)(n>1)$ are not isomorphic to $\mathcal{M}$.

Proof. Since $\mathcal{R}_{0}$ and $\mathcal{R}_{1}$ are type $I I_{1}$ factors and $p_{j} \in \mathcal{R}_{0} \cap \mathcal{R}_{1}$, then there are orthogonal projections

$$
\begin{aligned}
& e_{1}, \ldots, e_{m_{1}} ; e_{m_{1}+1}, \ldots, e_{m_{2}} ; \ldots ; e_{m_{s-1}+1}, \ldots, e_{m_{s}} \in \mathcal{R}_{0} \\
& f_{1}, \ldots, f_{m_{1}} ; f_{m_{1}+1}, \ldots, f_{m_{2}} ; \ldots ; f_{m_{s-1}+1}, \ldots, f_{m_{s}} \in \mathcal{R}_{1}
\end{aligned}
$$


such that $\tau\left(e_{j}\right)=\tau\left(f_{j}\right)=\frac{1}{k}\left(k=m_{s}\right)$ and

$$
\begin{aligned}
& e_{m_{j-1}+1}+\cdots+e_{m_{j}} \leq p_{j}<e_{m_{j-1}+1}+\cdots+e_{m_{j}}+e_{m_{j}+1}, \quad \forall 1 \leq j \leq s-1 \\
& f_{m_{j-1}+1}+\cdots+f_{m_{j}} \leq p_{j}<f_{m_{j-1}+1}+\cdots+f_{m_{j}}+f_{m_{j}+1}
\end{aligned}
$$

Since $\mathcal{R}_{0}$ and $\mathcal{R}_{1}$ are type $I I_{1}$ factors, then there exist unitary elements $u_{j} \in$ $\mathcal{R}_{0}, v_{j} \in \mathcal{R}_{1}$ and $w \in \mathcal{M}$ such that $u_{j} e_{1} u_{j}^{*}=e_{j}, v_{j} f_{1} v_{j}^{*}=f_{j}(j=1, \ldots, k)$ and $w e_{1} w^{*}=f_{1}$. So

$$
\begin{aligned}
M & =\overline{s p} \mathcal{R}_{0} \mathcal{R}_{1}=\overline{s p} \mathcal{R}_{0}\left(\sum e_{i}\right)\left(\sum f_{j}\right) \mathcal{R}_{1} w \\
& =\overline{s p}\left\{\mathcal{R}_{0} e_{i} f_{j} \mathcal{R}_{1} w ; m_{l} \leq i, j \leq m_{l+1}, 0 \leq l \leq s-1\right\} \\
& =\overline{s p}\left\{\mathcal{R}_{0} u_{i} e_{1} u_{i}^{*} v_{j} f_{j} v_{j}^{*} \mathcal{R}_{1} w ; m_{l} \leq i, j \leq m_{l+1}, 0 \leq l \leq s-1\right\} \\
& =\overline{s p}\left\{\mathcal{R}_{0} e_{1} u_{i}^{*} v_{j} f_{1} \mathcal{R}_{1} w ; m_{l} \leq i, j \leq m_{l+1}, 0 \leq l \leq s-1\right\} .
\end{aligned}
$$

Therefore,

$$
\begin{aligned}
e_{1} \mathcal{M} e_{1} & =\overline{s p}\left\{e_{1} \mathcal{R}_{0} e_{1} u_{i}^{*} v_{j} w e_{1} w^{*} \mathcal{R}_{1} w e_{1} ; m_{l} \leq i, j \leq m_{l+1}, 0 \leq l \leq s-1\right\} \\
& =\overline{s p}\left\{e_{1} \mathcal{R}_{0} e_{1} e_{1} u_{i}^{*} v_{j} w e_{1} e_{1} w^{*} \mathcal{R}_{1} w e_{1} ; m_{l} \leq i, j \leq m_{l+1}, 0 \leq l \leq s-1\right\} .
\end{aligned}
$$

Also by Proposition 2, we know that $e_{1} \mathcal{M} e_{1}$ is not isomorphic to any one of free group factors $L\left(F_{n}\right), n>2 \sum_{l=0}^{s-1}\left(m_{l+1}-m_{l}+1\right)^{2}+6$. We also know that $\frac{m_{l+1}-m_{l}+1}{k}=\tau\left(e_{m_{l}}+\cdots+e_{m_{l+1}}\right) \leq \tau\left(p_{l+1}\right)+\frac{2}{k}$. Then

$$
\begin{aligned}
& 6+2 \sum_{l=0}^{s-1}\left(m_{l+1}-m_{l}+1\right)^{2} \\
& \leq 6+2 \sum_{l=0}^{s-1}\left(k \tau\left(p_{l+1}\right)+2\right)^{2} \\
& \leq 6+2 \sum_{l}\left(k^{2} \tau\left(p_{l+1}\right)^{2}+4 k \tau\left(p_{l+1}\right)+4\right) \\
& \leq 6+2 a k^{2}+8 k+8 s .
\end{aligned}
$$

Therefore, free group factors $L\left(F_{n}\right), n>6+2 a k^{2}+8 k+8 s$, are not isomorphic to $e_{1} \mathcal{M} e_{1}$.

If free group factor $L\left(F_{2}\right)$ is isomorphic to $\mathcal{M}$, then $e_{1} \mathcal{M} e_{1}$ is isomorphic to free group factor $L\left(F_{k^{2}+1}\right)$ by Proposition 1 On the other hand, when $k$ is big enough, $k^{2}+1>6+2 a k^{2}+8 k+8 s$. From the above proof, the free group factor $L\left(F_{k^{2}+1}\right)$ is not isomorphic to $e_{1} \mathcal{M} e_{1}$. This is contradiction. So all free group factors $L\left(F_{n}\right)(n>1)$ are not isomorphic to $\mathcal{M}$.

\section{ACKNOWLEDGMENTS}

The authors are grateful to Liming Ge and Bingren Li for their support and helpful discussion.

\section{REFERENCES}

1. A. Connes and V. Jones, Property $\mathrm{T}$ for von Neumann algebras, Bull. London Math. Soc. 17(1985), 57-62. MR0766450 (86a:46083)

2. Liming Ge, Applications of free entropy to finite von Neumann algebras, Amer. J. Math. 119(1997), 467-485. MR.1439556 (98a:46074)

3. Liming Ge, Applications of free entropy to fnite von Neumann algebras, II, Ann. Math. 147 (1998), 143-157. MR1609522 (99c:46068)

4. Liming Ge and Sorin Popa, On some decomposition properties for factors of type $I I_{1}$, Duke Math, 94 (1998), 79-101. MR1635904 (99j:46070)

5. S. Popa, Symmetric enveloping algebras, amenability and AFD properties for subfactors, Math. Res. Lett. 1(1994), 409-425. MR.1302385 (95i:46095)

6. Marius B. Stefan, The indecomposability of free group factors over nonprime subfactors and abelian subalgebras, preprint. 
7. Dan Voiculescu, Circular and semicircular systems and free product fanctors, In: Operator Algebras, Unitary Representations, Enveloping Algebras and Invariant Theory (Prog. Math., vol. 92, pp. 45-60 Boston; Birkhauser 1990). MR1103585 (92e:46124)

8. Dan Voiculescu, The analogues of entropy and of Fisher's information measure in free probability theory, II, Invent Math. 118 (1994), 411-440. MR1296352 (96a:46117)

9. Dan Voiculescu, The analogues of entropy and of Fisher's information measure in free probability theory, III: The absence of Cartan sualgbras, Geom. Funct. Anal. 6 (1996), 172-199. MR1371236 (96m:46119)

Department of Mathematics, University of Pennsylvania, Philadelphia, Pennsylvania 19104-6395

E-mail address: junhao@math.upenn.edu

Department of Mathematics, University of Yan Tai, Shan Dong 264005, People's RePUBLIC OF CHINA

E-mail address: zxxmath@yahoo.com.cn 